\title{
RESEARCH ON THE KEY TECHNOLOGY OF LARGE SCALE MAPPING FROM LOW ALTITUDE PHOTOGRAMMETRY
}

\author{
Wu bo-yi ${ }^{\text {a }}$, Zhang Ning ${ }^{\text {b* * Su guo-zhong }}{ }^{\mathrm{c}}$ \\ ${ }^{a}$ Shanxi province Basic Geographic Information Institute, 030001Taiyuan China, boyiwu123@126.com \\ b China Land Surveying and Planning Institute, Ministry of Land and Resources, 100035 Beijing, $504110708 @ q q . c o m$ \\ ${ }^{\mathrm{c}}$ Chinese Academy of Surveying \& Mapping,100039 Beijing,174083588@qq.com
}

\section{Commission I, ICWG I/Vb}

KEY WORDS: Large-scale Photogrammetry, Wide-angle Camera, Elevation Accuracy, Low Altitude Photogrammetry Production Process, Low Altitude Photogrammetric DLG Production, Multi-center Image Projection Reconstruction

\begin{abstract}
:
Based on the theoretic analysis of the accuracy in large scale photogrammetric mapping, some defects in traditional procedure were discussed. A set of key technologies dedicate to accuracy improvement in low altitude photogrammetry were analyzed in detail, namely the utilization of wide angle camera and low altitude flight, enhancement in image matching, predesigned layout of Ground Control Points (GCPs) in field survey, optimization of adjustment model and improvement in map processing. Besides, a low altitude aerial unmanned airship system was established. Finally, successful implementation in 1:500 topographic mapping project in built-up areas of 30 counties in Shanxi Province proves the practicability and effectiveness of the proposed approaches.
\end{abstract}

\section{INTRODUCTION}

In Chinese national topographic map series, large scale refers to be scale of 1:500, 1:1000 and 1:2000, which means better accuracy in cartography and higher requirement in photogrammetry. However, problems in large scale topographic mapping with traditional photogrammetric technologies have

not been completely resolved yet in spite of decades of researches (Boa et al., 2008). Table 1 shows the accuracy control index in mapping process from Chinese national criteria of aerial photogrammetry mapping process.

\begin{tabular}{|l|c|c|c|c|c|c|c|}
\hline Terrain & Points & \multicolumn{3}{|c|}{ Planimetric tolerance $(\mathrm{mm})$} & \multicolumn{3}{|c|}{ Height tolerance $(\mathrm{m})$} \\
\cline { 3 - 8 } category & & $1: 500$ & $1: 1000$ & $1: 2000$ & $1: 500$ & $1: 1000$ & $1: 2000$ \\
\hline Flat area & Fundamental orientation point & - & 0.3 & 0.3 & - & - & - \\
& Extra control Point & - & 0.5 & 0.5 & - & - & - \\
& Common Point error & - & 0.8 & 0.8 & - & - & - \\
Hill area & Fundamental orientation point & - & 0.3 & 0.3 & - & 0.26 & 0.26 \\
& Extra control Point & - & 0.5 & 0.5 & - & 0.4 & 0.4 \\
Mountain & Common Point error & - & 0.8 & 0.8 & - & 0.7 & 0.7 \\
area & Fundamental orientation point & 0.4 & 0.4 & 0.4 & 0.26 & 0.4 & 0.6 \\
& Extra control Point & 0.7 & 0.7 & 0.7 & 0.4 & 0.6 & 1.0 \\
Alpine & Common Point error & 1.1 & 1.1 & 1.1 & 0.7 & 1.0 & 1.6 \\
area & Fundamental orientation point & 0.4 & 0.4 & 0.4 & 0.4 & 0.75 & 0.9 \\
& Extra control Point & 0.7 & 0.7 & 0.7 & 0.6 & 1.2 & 1.5 \\
& Common Point error & 1.1 & 1.1 & 1.1 & 1.0 & 2.0 & 2.4 \\
\hline
\end{tabular}

Table 1. Accuracy control index in mapping process

As shown in Table 1, accuracies of 1:500 aerial photogrammetric mapping in Chinese national criteria in flat and hill areas are not clear. This indicates it's difficult to reach that high precision requirement with aerial photogrammetric technology. Furthermore, specific demands for large scale mapping are as follows: In aforementioned mapping area where the aerial photogrammetry technology is not suitable, elevation points must be acquired with full field survey method, which means every $100 \mathrm{~cm}^{2}$ district on map should include 10 to 20 points in flat and hill areas or 8 to 15 points in mountain and
${ }^{1}$ alpine areas and other region with scarce terrain feature points. This fully demonstrates the aerial photogrammetry technology in large scale topographic mapping has many deficiencies.

The main goal of this paper is to introduce a set of key technologies to solve aforementioned problems with modern low altitude photogrammetric technology.

${ }^{*}$ Corresponding author 


\section{KEY TECHNOLOGY IN LARGE SCALE TOPOGRAPHIC MAPPING WITH AREIAL POTOGRAMMTRY}

The method using planimetric and elevation data from aerial photography measuring instrument to produce topographic map is called universal aerial photogrammetric mapping (UAPM). In order to make this method meet the accuracy and efficiency requirements, measurements such as photogrammetric camera selection, flight pattern, aerial triangulation and digital line graphic (DLG) production are involved.

\subsection{Wide Angle Camera}

Field angle of aerial camera has a great influence on the accuracy of aerial photogrammetry, no matter it is used in relative orientation equation or collinearity equation for exterior orientation elements. This theory has been proved by many researchers in different projects. Table 2 shows the standard deviation of every relative orientation elements with different field angles (Lin et al., 2008; Lin et al., 2014a).

\begin{tabular}{|l|c|c|c|}
\hline Field angle $(2 \theta)$ & $\sigma_{\varphi}=\sigma_{\varphi^{\prime}}$ & $\sigma_{\omega^{\prime}}$ & $\sigma_{\kappa}=\sigma_{\kappa^{\prime}}$ \\
\hline $26^{\circ}$ & $0.0042^{\circ}$ & $0.0051^{\circ}$ & $0.0230^{\circ}$ \\
$38^{\circ}$ & $0.0032^{\circ}$ & $0.0038^{\circ}$ & $0.0138^{\circ}$ \\
$53^{\circ}$ & $0.0022^{\circ}$ & $0.0026^{\circ}$ & $0.0065^{\circ}$ \\
$74^{\circ}$ & $0.0015^{\circ}$ & $0.0018^{\circ}$ & $0.0033^{\circ}$ \\
$90^{\circ}$ & $0.0010^{\circ}$ & $0.0012^{\circ}$ & $0.0026^{\circ}$ \\
\hline
\end{tabular}

Table 2. Standard deviation of relative orientation elements with different field angles

From Table 2, conclusion can be drawn as follows: When long focus camera is used in high altitude aerial photogrammetry, due to the limited accuracy of exterior orientation elements, positioning and orientation system (POS) with high accuracy and stable platform should be employed; however, the precision of aerial triangulation can be improved with wide angle camera in low altitude photogrammetry. In addition, combined wideangle camera with small volume and light weight should be a prior choice especially in low altitude photogrammetry with light and small unmanned aerial vehicle (UAV) (Zhang Y. et al., 2008a; Hai et al., 2010a; Robert et al., 2011a).

\subsection{Low altitude photogrammetry}

Acquisition for high resolution image is the basis for high precision aerial photogrammetric mapping. However, low altitude photogrammetry has special demands for successful application conditions as follows: Aircraft should be safe enough and hardware for data storage for camera exposure and flight route for aerial photogrammetric stereo imaging should be designed carefully to meet the requirement. Since low altitude photogrammetry has special features such as scattered distribution and complex terrain, particular software for flight route plan namely NavGT is developed. DEM data from NAS can reach height accuracy as high as $10 \mathrm{~m}$ within 1 second or even better, which can support for flight route plan wherever in China. NavGT can automatically produce flight routes with $60 \%$ endlap and $30 \%$ slidelap in assigned area. Meanwhile, these routes can meet the requirement of camera exposure parameters and safety flight altitude. Furthermore, unlike traditional high altitude photogrammetry, the widths of these routes are unequal. As shown in Figure 1, space between two flight route in urban area of Yongji county in Yuncheng City are changeable in accordance with terrain, in addition, list on the left shows the height of each flight rout is not equal, either.

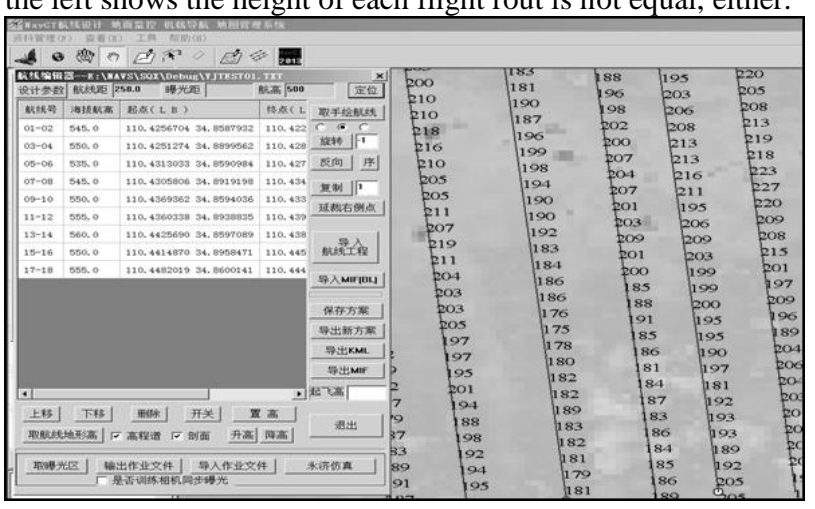

Figure 1. Airline created by Nav GT based on countrywide DEM.

Moreover, the geometric accuracy in low altitude photogrammetry can be improved by making full use of the priority of wide angle camera and precise driving performance of UAV as well as applying special photogrammetry method such as increasing flight overlap and designing routes accommodate to terrain. If weather and geography conditions permit, predefined flight routes must be strictly followed, for it means a lot to the edge area photogrammetric accuracy.

\subsection{Aerial triangulation accuracy}

To apply universal aerial photogrammetric mapping successfully in large scale topographic mapping, namely to get all detail elevation points of terrain line map from aerial photogrammetric stereo models, there has to be relevant aerial triangulation results with demanded accuracy to ensure that the geometric precision of each 3D model can meet the mapping requirements. Theoretically, aerial triangulation accuracy includes two aspects. On one hand, field angle of photogrammetric camera, flight baseline to height ratio, image resolution and the measurement accuracy of image control points should be taken into consideration in field survey. On the other hand, the precision of camera distortion model, the reliability and the veracity of image matching and weighted adjustment model of whole network must be chosen carefully in indoor operation. Those factors are relevant to each other. Restricted by the development of technology, there are technical bottleneck in large scale topographic mapping with traditional photogrammetry all the time. Thus, the combination of wide angle camera and low altitude aircraft provides a good opportunity to solve those problems in traditional photogrammetry. In this paper, four pairs of combined wide angle camera with $80^{\circ} \times 86^{\circ}$ field angle work above $200 \mathrm{~m}$ to $500 \mathrm{~m}$ high in the air, with successful acquisition of wide angle, big flight baseline to height ratio (theoretically 0.9) and high resolution (better than $0.05 \mathrm{~m}$ ) demanded in large scale photogrammetry. Finally, The mean square error of planimetric and height control points less than $10 \mathrm{~cm}$ and mean square error of model connection points is better than aerial triangulation results $(15 \mathrm{~cm})$ after series of key technologies. Those methods make it possible to use universal aerial photogrammetric mapping in large scale photogrammetry with accuracy better than $5 \mathrm{~cm}, 10 \mathrm{~cm}$ and $15 \mathrm{~cm}$ in 1:500, 1:1000 and 1:2000 topographic map production, respectively. 
2.3.1 POS: In low altitude photogrammetry, the most common equipment is cheap and simple POS. Although precision of POS is quite low $\left( \pm 1 \mathrm{~m}\right.$ in GPS precision and $\pm 2^{\circ}$ in pose accuracy), it is absolutely useful in indoor operation. First of all, it can be used to sort the aerial photographs automatically; then, it can offer approximate orientation elements in aerial triangulation adjustment; at last, it can restrain the gross error in bundle adjustment to improve the accuracy of the results.

2.3.2 Image matching: Imaging matching has been researched for decades in aerial photogrammetry (Zhang Z. et al., 2004a; Zhang Y. et al.,2011a) with slow development. But in recent years, new technology such as computer technology has promoted new breakthroughs with unbelievable speed. The typical theory of image matching must be SIFT with geometric feature invariant as theoretical foundation (David G. Lowe, 1999, 2004a). This theory has brought new breakthrough in homonymy points matching with multi-view images. Methods referred in this paper are on the basis of SIFT with two improvements as follows: Parallel processing of computers is utilized to optimize the algorithm of feature description and feature matching, besides, adaptive windows with size of $17 \times 17$ to $129 \times 129$ are adopted to reduce the matching errors and guarantee accuracy of sub pixel of match points; iterative matching strategy RANSC(Fischler et al.,1981a) is used to eliminate gross error points and interpolate encrypted dots to ensure the total error rate of homonymous points matching under $5 \%$.

2.3.3 Accuracies of control points: According to "GB/T79312008 ", strict requirements about how to arrange the control point location and how to choose instrument and method are demonstrated in detail, with basic principle of control points in field survey better than 1 pixel (GSD). It means planimetric and height accuracies of control points in 1:500 aerial photogrammetric surveys should be better than $5 \mathrm{~cm}$, which can be satisfied by GPS-RTK or GPS-CORS technology. However, traditional measurement of control points has three drawbacks. First, the operation period is too long. Field survey should be carried out with rough ortho-images and control images after the flight finished. Secondly, it is difficult to match the optimal control points position in field survey with obvious feature points on images, for the selection of feature points is conducted after the field survey. At last, in order to improve the height accuracy of image control network, control points should be better distributed in plat area where the feature points with high planimetric location accuracy is hard to find due to limited texture.

In low altitude photogrammetry, predesigned layout of control points before the flight can reduce the aerial photogrammetric production time and improve the accuracy and quality of control points. The control points without geometric distortion should be distributed in flat area with good visibility conditions in advance. Large quantities of experiments show that the universal aerial photogrammetric mapping can completely satisfied the accuracy requirement of aerial triangulation results in 1:500 topographic mapping if methods proposed in this paper are used as follows: Control points should be layout with approximately equal width in survey area; control point groups each with four control points should be located on four corners of the survey area; moreover, predesigned flight routes and MAPATM models should be employed.
Well-designed control points can improve measurement accuracy. Due to the interaction of imaging spectrometer, there are special phenomena called "black corrosion by white" on colorful digital images. As shown in Figure 3, black marker Y with equal width gradually narrows visually and the white block seems bigger than the black one in spite of the same size $(20 \mathrm{~cm}$ $\times 20 \mathrm{~cm}$ ). This phenomenon is common in real world. Thus color difference will not be the only factor that needs to be considered when choosing control points . On account of this spectral characteristic, control points with high precision are designed as shown in Figure 2. The principle of this proposed approach is to extract the edge line by using interactional color stripes and get the barycentric coordinates of error triangle from edge lines to replace image intersection coordinates of edge line, which can guarantee the control points accuracy to meet the subpixel level.

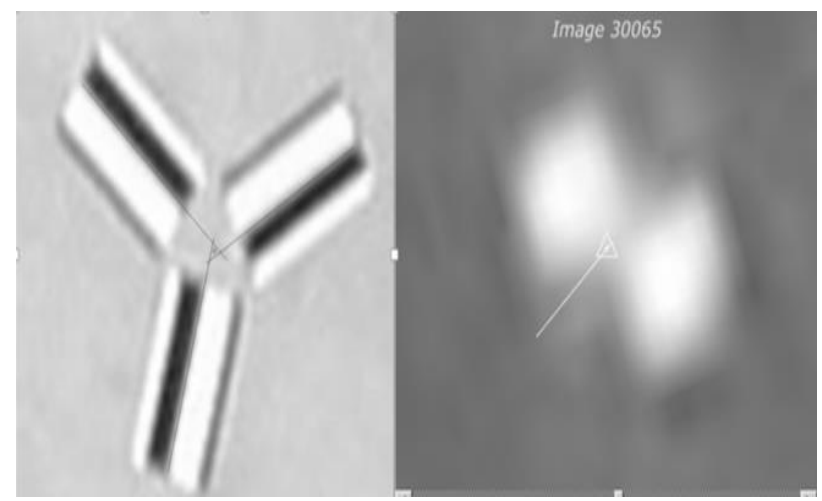

Figure 2. Schematic diagram of improving the image point accuracy in measurement

2.3.4 Adjustment model: There are several famous softwares for aerial triangulation in traditional photogrammetry, such as PATB, INPHO, BINGO, Orima and SSK, all of which use bundle adjustment. However, exterior orientation elements can be obviously different when different softwares are employed to calculate their values despite of using the same aerial photogrammetric data and ground control materials. The reason should be that the process of the image reconstruction from $2 \mathrm{D}$ to $3 \mathrm{D}$ is a pathological one. Furthermore, influenced by the various errors and mismatches, aerial triangulation network is a soft network formed by the lines generated by "main pointsimage points-ground points" process. Thus different sensors with different adjustment models according to various understanding and requirements are designed and adopted to make aerial triangulation network more realistic for higher accuracy.

In order to solve height accuracy problems in large scale photogrammetry and potential matters without field work for height points in 1:500 aerial photogrammetric mapping, several methods are improved.

1) The feature properties of wide angle image are used to get exterior orientation elements and diagonal elements, meanwhile, the overlap degree of each pair of match points are checked, furthermore, weighted structure network should be built according to different models' intersection angles.

2) The utilization of POS data improves overall aerial triangulation accuracy a lot, however, the exposure position data contains obvious systematic and random errors due to 
synchronization exposure, dynamic and differential GPS analysis, projection transformation and equipment errors, etc. Thus, strict static model of POS and camera geometric position should be established while dynamic weight method in aerial triangulation adjustment process should be employed to make the network with reasonable convergence.

3) Since multi-view image matching process is a pathological one, situations of mismatching and unequal precision matching are inevitable. To solve this problem, increasing the density of matching points can reduce mismatch probability and RANSC method can remove blunders for better control network adjustment precision.

4) To improve physical stiffness of aerial triangulation, different measurements namely advanced layout of flight routes, reasonable selection of control points, inhibition of endlap and slidelap overlap and increment of flight lines are adopted.

\subsection{Aerial photogrammetric products}

Compared with traditional film photography, the proposed method requires more computer resources, for it needs 4 stereo models which contain 6 wide-angle images and 24 sub-images to produce 1 image with the utilization of $80^{\circ} \times 86^{\circ}$ wide angle camera in 1:500 low altitude photogrammetry mapping. Fortunately, the development of computer technology can provide abundant computing resources for low altitude photogrammetry. At the same time, low altitude photogrammetry can offer a large quantity of geographic information products for the information age, as shown in Figure 3.

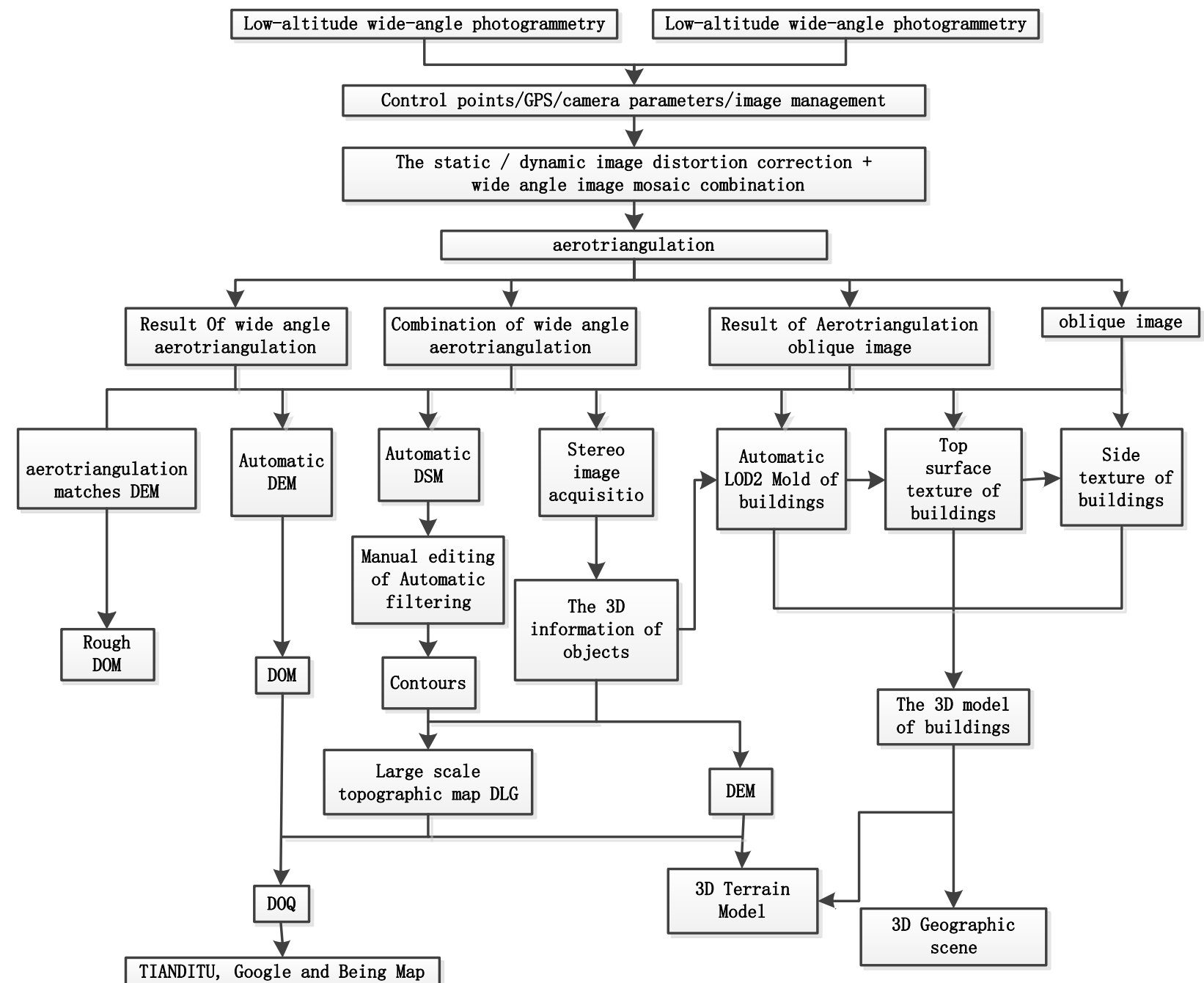

Figure 3. Production process of low altitude and large scale photogrammetry products

Once aerial triangulation is finished with pretreatment of low altitude photogrammetry and field survey data, four kinds of mapping products namely digital elevation model (DEM), digital orthophoto map (DOM), topographic contour (TC) and digital line graph (DLG) can be produced.

DEM data with different accuracies can be output during any procedure in low altitude photogrammetry. Rough DEM can be produced with the utilization of high accuracy match points from aerial triangulation. Dense point cloud can be generated by using aerial triangulation products and wide angle images and then DEM can be interpolated. In addition, interpolated DEM can be constrained by contour lines, 3D information of ground features and discrete 3D points. The former two kinds of DEM can satisfy different application requirements while the latter one belongs to be standard production. 
DOM can be output by using relevant DEM, wide angle images and aerial triangulation products in accordance with different efficiencies and accuracies.

TC can be generated by DSM method with dense point cloud data, filter approach with terrain surface triangulation and interpolated way with contour line in large scale mapping. However, terrain contour may be better collected by manual way from stereoplotter in small scale topographic mapping.

In order to ensure that low altitude photogrammetry has the same efficiency with high altitude photogrammetry in data acquisition from stereoplotter, five aspects of technologies have been improved by taking advantage of computer science. A) Unlike traditional topographic mapping, the generation of epipolar line can be avoided if real-time epipolar lines are used to generated stereo model and manual stereoscopic acquisition is conducted after aerial triangulation. B) Without concern for the switching of a single stereo model in topographic mapping, the stereo model designed in this paper can be switched automatically to present stereo scene of survey area. C) Since high resolution model means high efficiency, dynamic stereo model can be constructed, which ensures the measuring mark with high resolution no matter which part it belongs to on the large altitude difference model, furthermore, the construction of sub-pixel level stereo model can improve local measurement accuracy. D) In accordance with the latest national mapping criteria, all collected ground features should contain 3D information and automatically convert to $2 \mathrm{D}$ symbols for standard topographic map generation, which means field survey should be in consistent with map compilation. E) For productivity improvement, technologies such as GPU, multicore CPU, internet and disk array storage are employed in large scale aerial photogrammetry.

In a nutshell, large scale aerial photogrammetry can be combined with oblique photograph. By using wide angle camera in low altitude flight, even one flight can produce 1:500 DLG images as well as slop textures of 3D model of buildings. This approach can save airspace resource and production costs of geographic information at the same time.

\section{EXPERIENT AND RESULT}

\subsection{Low altitude aerial unmanned airship system}

With the utilization of low altitude aerial unmanned airship system after one year' research, the production task of large scale topographic mapping with low altitude photogrammetry in built-up area of 30 counties in Shanxi province was completed successfully.

Airship is the most secure aircraft in city's low altitude aerial survey. The low altitude aerial unmanned airship consists of four parts, namely airship platform, mobile operation support system, combined wide angle camera and software consistent with aerial photogrammetry. As shown in Figure 4, airship platform is composed of airbag, power, bridge equipment, pressure and sash, meanwhile, mobile operation support system consists of helium recovery system, mobile boathouse and transport truck.

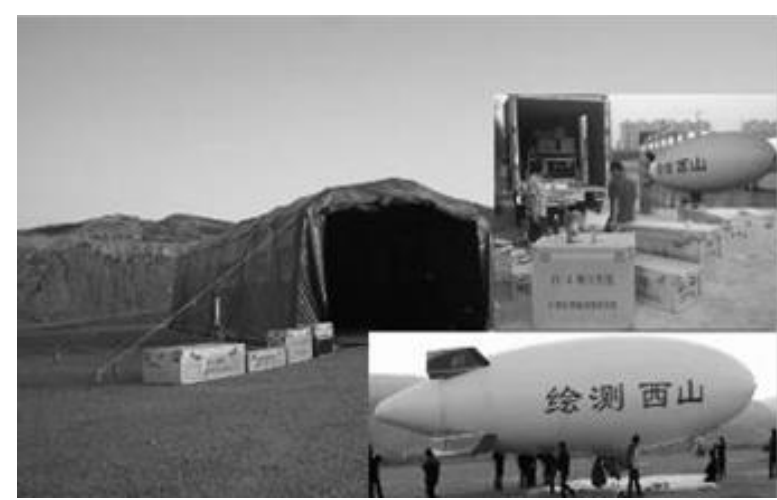

Figure 4. low altitude aerial unmanned airship system

\subsection{Combined wide angle camera}

As shown in Figure 5, the combined wide angle camera includes six parts, namely a multi-unit optical imaging system, a combined mechanical component, a imaging control system, a secondary image computing unit, a three-axis stabilized platform and a differential GPS.

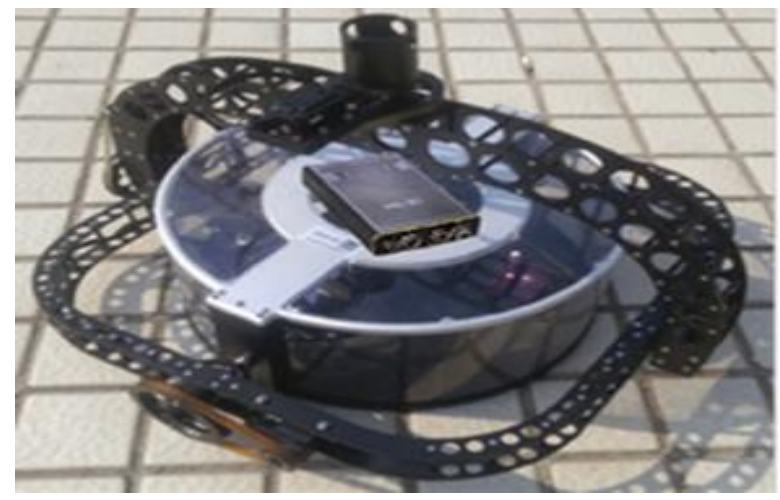

Figure 5. Combined wide angle aerial camera

There are two methods for guaranteeing mapping accuracy adopted by the combined wide angle camera, namely static ground calibration and dynamic flight calibration. Figure 6 shows the focal length (f) and main point $\left(\mathrm{x}_{0}, \mathrm{y}_{0}\right)$ of calibrated camera for large depth field. Short distance calibration in indoor is to find out the low level distortion of camera while long range calibration in outdoor is to detect camera's high level distortion.

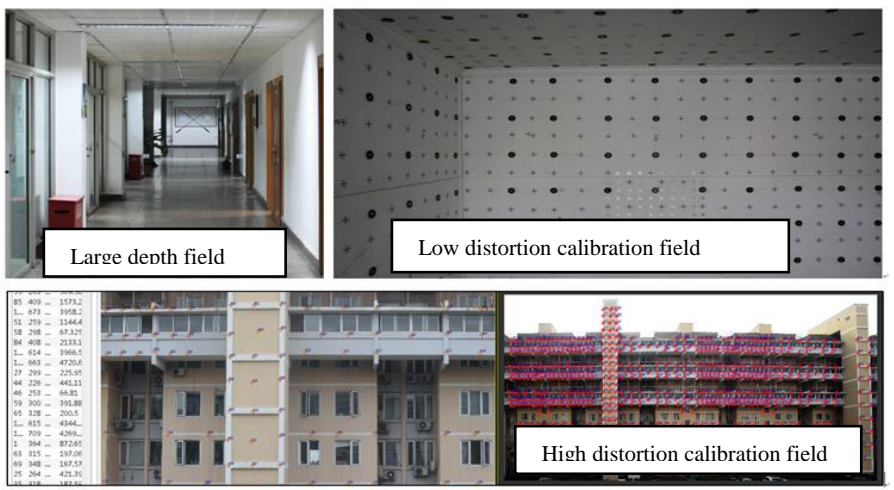

Figure 6. The camera calibration field 
The proposed comprehensive camera calibration mode could eliminate the interaction influence caused by external and interior orientation elements when distortion model parameters are calculated. Thus the geometric precision of aerial images was improved. In addition, parts of control points' absolute errors in comprehensive calibration are demonstrated in Table 5.

\begin{tabular}{|l|c|c|c|c|c|c|c|c|c|c|c|}
\hline NO. & $\Delta \mathrm{X}$ & $\Delta \mathrm{Y}$ & $\mathrm{NO}$. & $\Delta \mathrm{X}$ & $\Delta \mathrm{Y}$ & $\mathrm{NO}$. & $\Delta \mathrm{X}$ & $\Delta \mathrm{Y}$ & $\mathrm{NO}$. & $\Delta \mathrm{X}$ & $\Delta \mathrm{Y}$ \\
\hline 171 & -0.01 & 0.01 & 293 & -0.02 & 0.00 & 314 & -0.01 & -0.06 & 334 & 0.00 & -0.01 \\
182 & -0.06 & 0.01 & 294 & -0.04 & 0.01 & 315 & -0.02 & -0.05 & 335 & -0.01 & -0.07 \\
184 & -0.03 & -0.02 & 295 & -0.04 & -0.02 & 316 & 0.02 & -0.06 & 336 & 0.00 & -0.07 \\
185 & -0.05 & 0.00 & 296 & -0.01 & -0.03 & 317 & 0.02 & -0.05 & 337 & -0.03 & -0.03 \\
188 & -0.06 & 0.02 & 297 & 0.01 & -0.04 & 318 & -0.01 & 0.01 & 338 & -0.07 & -0.04 \\
190 & -0.06 & -0.01 & 298 & -0.06 & 0.00 & 319 & -0.02 & -0.07 & 339 & 0.00 & 0.00 \\
193 & -0.06 & -0.02 & 299 & -0.05 & 0.00 & 320 & -0.01 & -0.05 & 340 & -0.01 & -0.02 \\
194 & -0.04 & -0.02 & 300 & -0.03 & 0.00 & 321 & 0.01 & -0.04 & 341 & 0.02 & 0.01 \\
195 & -0.01 & 0.02 & 301 & -0.05 & 0.02 & 322 & -0.01 & -0.03 & 342 & -0.04 & -0.01 \\
197 & 0.02 & -0.02 & 302 & -0.06 & -0.05 & 323 & 0.02 & -0.03 & 343 & 0.01 & -0.03 \\
198 & -0.06 & 0.01 & 303 & -0.02 & -0.05 & 324 & 0.00 & -0.04 & 344 & -0.02 & -0.02 \\
201 & -0.03 & 0.00 & 304 & -0.01 & 0.02 & 325 & 0.01 & 0.00 & 345 & -0.01 & -0.07 \\
264 & -0.02 & -0.02 & 305 & 0.02 & -0.07 & 326 & 0.02 & -0.02 & 346 & 0.01 & -0.04 \\
266 & -0.01 & -0.03 & 306 & -0.02 & 0.00 & 327 & 0.00 & -0.04 & 347 & 0.02 & 0.00 \\
267 & -0.04 & 0.02 & 307 & -0.03 & 0.00 & 328 & -0.04 & 0.02 & 348 & 0.01 & -0.07 \\
278 & 0.02 & -0.04 & 308 & 0.00 & -0.04 & 329 & -0.07 & -0.02 & 349 & -0.05 & 0.02 \\
288 & 0.00 & -0.03 & 309 & -0.04 & 0.02 & 330 & -0.01 & 0.02 & 350 & -0.03 & 0.02 \\
290 & -0.06 & 0.01 & 310 & 0.00 & -0.01 & 331 & -0.02 & -0.04 & 351 & 0.00 & -0.06 \\
291 & -0.01 & -0.03 & 311 & 0.02 & -0.03 & 332 & -0.04 & -0.01 & 352 & 0.00 & -0.06 \\
292 & 0.01 & 0.26 & 312 & -0.04 & 0.01 & 333 & -0.01 & -0.07 & 353 & -0.05 & 0.00 \\
\hline
\end{tabular}

Table 5. Absolute error of ground static calibration model

\subsection{Experiment}

The Designed experiment area was $25 \mathrm{~km}^{2}$ in urban area of Wanrong County, including $7.5 \mathrm{~km}^{2}$ center areas for 1: 500 fundamental topographic mapping and $17 \mathrm{~km}^{2}$ built-up areas for 1: 1000 fundamental topographic mapping. Wide angle camera LAC04 was employed, parameters of which are shown in Table 6.

\begin{tabular}{|l|c|}
\hline Imaging system index \\
\hline Focal length & $35 \mathrm{~mm}$ \\
Pixel & $6.4 \mathrm{um}$ \\
Total field angle & $80^{\circ} \times 70^{\circ}$ \\
Image size & $7165 \times 9024$ \\
Minimum interval & $2.0 \mathrm{~s}$ \\
Weight & $15 \mathrm{~kg}$ \\
\hline Work condition index \\
\hline Flight altitude & $150 \sim 500 \mathrm{~m}$ \\
Flight speed & $30 \sim 60 \mathrm{~km} / \mathrm{h}$ \\
Work wind level & $0-3$ \\
Maximum wind level & 5 \\
Temperature & $-10 \sim 40{ }^{\circ} \mathrm{C}$ \\
Humidity & $65 \%$ \\
\hline
\end{tabular}

Table 6. Parameters of wide angle camera( LAC04)

The flight height in 1:500 topographic mapping was $180 \mathrm{~m}$ to $230 \mathrm{~m}$ while it was $300 \mathrm{~m}$ to $350 \mathrm{~m}$ in 1: 1000 topographic mapping. Parts of exposure points are presented in Figure 7, which were designed according to the principles of predefined and autonomous low altitude flight route. Based on the principles of photogrammetric network arrangement, layout of the control points was designed as follows: Boundary interval distance was about 300 meters along the route direction while middle interval distance was approximate 500 meters, so 108 height control points and 200 checkpoints were distributed in $10 \mathrm{~km}^{2}$ area in 1: 500 topographic mapping. Furthermore, aerial triangulation with constraint of GPS, framework and wide angle images were adopted to make all checkpoints meet the required accuracy_planimetric mean square error within $0.08 \mathrm{~m}$ and planimetric maximum absolute difference within $0.24 \mathrm{~m}$ while the height mean square error within $0.07 \mathrm{~m}$ and height maximum absolute difference within $0.18 \mathrm{~m}$.

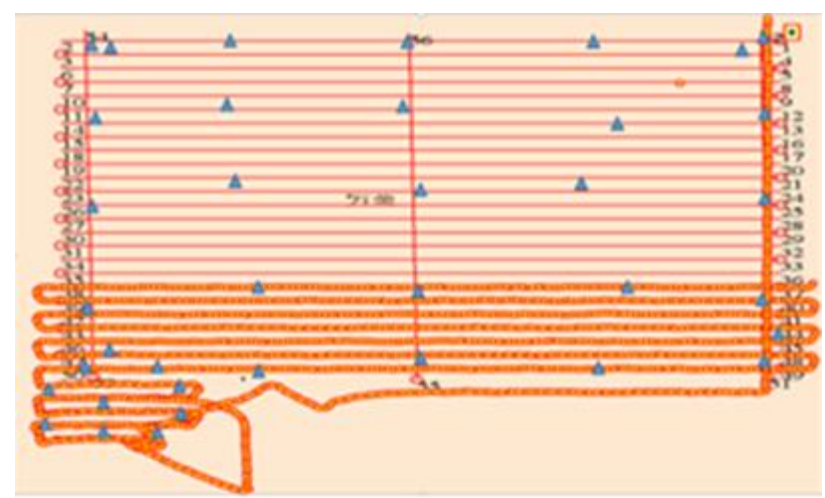

Figure 7.The control strip and flight trace 

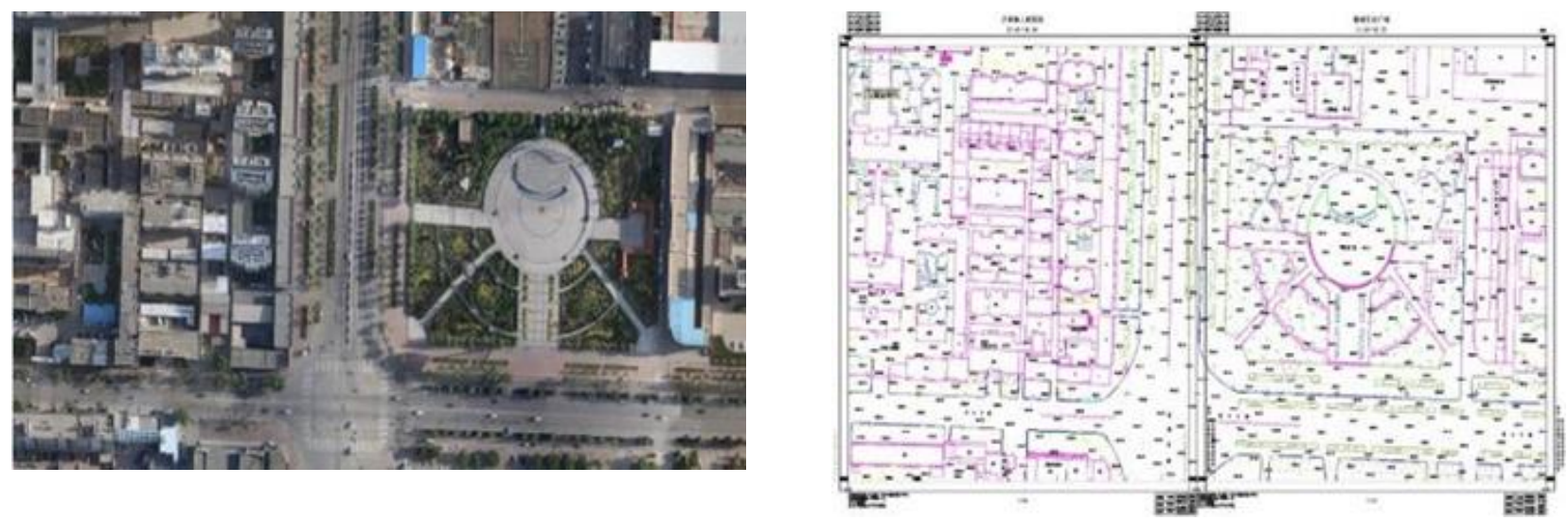

Figure 8. 1:500 DOM and DLG in study area

By using universal aerial photogrammetric mapping method in 1:500 topographic map, the production of DOM and DLG are presented in Figure 8, which have admitted by the quality inspection departments of surveying and mapping industry. In addition, comprehensive scoring method and the scores of field points selected from 1: 500 DLG products of Wanrong county in Yuncheng city are shown in Table 7.

\begin{tabular}{|c|c|c|c|c|c|c|}
\hline Image number & $\begin{array}{c}\text { Mathematical } \\
\text { accuracy } \\
(0.2)\end{array}$ & $\begin{array}{c}\text { Data and } \\
\text { structure } \\
(0.2)\end{array}$ & $\begin{array}{c}\text { Geographical } \\
\text { accuracy } \\
(0.4)\end{array}$ & $\begin{array}{c}\text { Graphic quality } \\
(0.1)\end{array}$ & $\begin{array}{c}\text { Appendix } \\
\text { quality } \\
(0.1)\end{array}$ & $\begin{array}{l}\text { Sample } \\
\text { quality }\end{array}$ \\
\hline $05.25-99.50$ & 89 & 90 & 94 & 100 & 90 & 92.4 \\
\hline $05.50-99.50$ & 92 & 90 & 88 & 100 & 90 & 90.6 \\
\hline $05.50-99.75$ & 87 & 90 & 91 & 100 & 90 & 90.8 \\
\hline $05.75-99.50$ & 90 & 90 & 95 & 100 & 90 & 93 \\
\hline $05.75-99.75$ & 88 & 90 & 92 & 100 & 90 & 91.4 \\
\hline $06.00-99.50$ & 85 & 90 & 88 & 100 & 90 & 89.2 \\
\hline $06.25-99.50$ & 91 & 90 & 97 & 100 & 90 & 94 \\
\hline $06.50-99.50$ & 93 & 90 & 90 & 100 & 90 & 91.6 \\
\hline $06.75-99.50$ & 86 & 90 & 89 & 100 & 90 & 89.8 \\
\hline $07.25-00.00$ & 85 & 90 & 88 & 100 & 90 & 89.2 \\
\hline $07.25-99.00$ & 88 & 90 & 92 & 100 & 90 & 91.4 \\
\hline $07.25-99.25$ & 94 & 90 & 91 & 100 & 90 & 92.2 \\
\hline $07.25-99.50$ & 90 & 87 & 95 & 100 & 90 & 92.4 \\
\hline $07.25-99.75$ & 91 & 90 & 87 & 100 & 90 & 90 \\
\hline $07.50-99.00$ & 95 & 90 & 93 & 100 & 90 & 93.2 \\
\hline 07.75-98.75 & 87 & 90 & 91 & 100 & 90 & 90.8 \\
\hline $07.75-99.00$ & 86 & 90 & 89 & 100 & 90 & 89.8 \\
\hline $07.75-99.25$ & 92 & 90 & 88 & 100 & 90 & 90.6 \\
\hline $07.75-99.50$ & 88 & 90 & 92 & 100 & 90 & 91.4 \\
\hline 07.75-99.75 & 88 & 90 & 92 & 100 & 90 & 91.4 \\
\hline 08.00-00.00 & 90 & 86 & 95 & 100 & 90 & 92.2 \\
\hline $08.00-98.75$ & 89 & 90 & 94 & 100 & 90 & 92.4 \\
\hline $08.00-99.00$ & 90 & 90 & 85 & 100 & 90 & 89 \\
\hline $08.00-99.25$ & 86 & 90 & 89 & 100 & 90 & 89.8 \\
\hline $08.00-99.75$ & 92 & 90 & 88 & 100 & 90 & 90.6 \\
\hline $08.50-98.25$ & 93 & 90 & 90 & 100 & 90 & 91.6 \\
\hline $08.50-98.50$ & 90 & 90 & 85 & 100 & 90 & 89 \\
\hline 08.50-98.75 & 89 & 90 & 89 & 100 & 90 & 90.4 \\
\hline $08.75-98.50$ & 87 & 90 & 91 & 100 & 90 & 90.8 \\
\hline $08.75-98.25$ & 88 & 89 & 92 & 100 & 90 & 91.2 \\
\hline
\end{tabular}

Table 7. Product sampling and testing score of 1:500 DLG in study area 


\section{CONCLUSIONS}

The theoretical analysis and the experiment with the utilization of unmanned airship carrying combined wide angle camera in one year's practical implement in Shanxi Province demonstrates that the proposed approaches are efficient. Using low altitude photogrammetry to realize large scale mapping is entirely possible if key methods such as selection of wide angle camera and low altitude flight, enhancement in image matching, predesigned layout of Ground Control Points (GCPs), optimization of adjustment model and improvement in map processing are conducted. It is hopeful that it will become national standard in large scale topographic mapping if more practice and promotion are conducted in more places with low altitude aerial photogrammetry technology.

\section{ACKNOWLEDGEMENTS}

This work was supported by Project of Comprehensive Detection Technology and Interpretation System of Airborne Geophysical and Remote Sensing with project number 2013AA063905, which belongs to The National High Technology Research and Development Program of China (863).

\section{REFERENCES}

Boa ping, L., Xinpu, S., Ahiyu, X., Chengwen, E. and Bing, L., 2008. Actualize of Low Altitude Large Scale Aerophotography and Geodesic base on Fixed-wing Unmanned Aerial Vehicle Platform. In: The International Archives of the Photogrammetry, Remote Sensing and Spatial Information Sciences, Beijing, China, Vol. XXXVII, Part B1,pp. 643-646.

David G. Lowe, 1999.Object recognition from local scaleinvariant features. In: International Conference on Computer Vision, Corfu, Greece, pp. 1150-1157.

David G. Lowe, 2004a.Distinctive image features from scaleinvariant keypoints. International Journal of Computer Vision, 60, 2 (2004), pp. 91-110.

Hai, Y. C., Yong, C. C. and Yang, Q. C., 2010a. Autopilots for Small Unmanned Aerial Vehicles: A Survey. International Journal of Control, Automation and Systems, 8(1), pp.36-44.

Lin zongjian, 2008. UAV For Mapping-Low Altitude Photogrammetry Survey. International Society of Photogrammetry and Remote Sensing, 2008, pp.1183-1186

Lin zongjian, Xie feifei, Su guozhong, 2014a. Accuracy Analysis of Low Altitude Photogrammetry with Wide-angle Camera. Acta Geodaetica et Cartographica Scinica, 43(10), pp.991-996.

MA Fischler,RC Bolles,1981a. Random Sample Consensus: A Paradigm for Model Fitting with Applications to Image Analysis and Automated Cartography. Communications of the Acm, 24(6),pp.381-395.

Robert, P. B. and Maxine, E. D., 2011a. Evaluation of Bare Ground on Rangelands using Unmanned Aerial Vehicles: A case study. GIS Science \& Remote Sensing, 48, No.1, pp.74-85.

Zhang,Y., 2008a. Photogrammetric processing of low altitude image sequences by unmanned airship. International Archives of the Photogrammetry,Remote Sensing and Spatial Information Sciences, 37(B5), pp.751-758.

Zhang,Y., 2011a. Photogrammetric processing of low-altitude images acquired by unpiloted aerial vehicles. The Photogrammetric Record, 26(134), pp.190-211.

Zhang Z., 2004a. Aspects on aerial digital cameras. Engineering of surveying and mapping, 2004,13(2),pp.1-5. 